\title{
Stochastic Optimal Tracking with Preview for Linear Discrete-Time Markovian Jump Systems
}

\author{
Gou Nakura \\ Dept. of Engineering, Osaka University \\ 2-1 Yamadaoka, Suita, Osaka 565-0871, Japan \\ E-mail: nakura@watt.mech.eng.osaka-u.ac.jp
}

\begin{abstract}
In this paper we study the stochastic optimal tracking problems with preview for a class of linear discrete-time Markovian jump systems. The systems are described by the discrete-time switching systems with Markovian mode transitions. The necessary and sufficient conditions for the solvability of our optimal tracking problems are given by coupled Riccati difference equations with terminal conditions. Correspondingly feedforward compensators introducing future information are given by coupled difference equations with terminal conditions. We consider three different tracking problems depending on the property of the reference signals. Finally we give numerical examples.

Key Words: Markovian jump systems; Stochastic linear quadratic (LQ) optimization; Tracking control with preview; Coupled Riccati difference equations; Coupled feedforward compensators
\end{abstract}

\section{Introduction}

It is well known that, for design of tracking control systems, preview information of reference signals is very useful for improving performance of closed-loop systems, and recently much work has been done for preview control systems $([2,8,7,11,12,13,14,15,16,17])$. Especially, in order to design tracking control systems for a class of systems with rapid or abrupt changes, it is effective in improving tracking performance to construct control systems considering future information of reference signals.

In the cases with no mode transitions, U. Shaked and C. E. de Souza ([17]) have studied the $\mathrm{H}_{\infty}$ tracking control theory with preview for continuous-time linear time-varying systems by a game theoretic approach, and, A. Cohen and U. Shaked ([2]) have also extended their theory for discrete-time linear systems. Recently the author has extended their theory to linear impulsive systems $([13,14])$. It is very important to consider effects of stochastic noise or uncertainties for tracking control systems, and so, by Gershon et al., the theory of stochastic $\mathrm{H}_{\infty}$ tracking with preview has been presented for linear continuous- and discrete-time systems respectively $([8,7])$.
Markovian jump systems ([1, 3, 4, 5, 6, 10, 18, 19, $20,21])$ have abrupt random mode changes in their dynamics. The mode changes follow Markov processes. Such systems may be found in the area of power systems, manufacturing systems, communications, aerospace systems, financial engineering and so on. Such systems are classified into continuous-time $([1,10,18,19,20,21])$ and discrete-time $([3,4,5,6])$ systems. The optimal and $\mathrm{H}_{\infty}$ control theory has been presented for each of these systems respectively $([20,21],[18],[3,5],[6])$. Recently the author has presented the LQ, LQG and $H_{\infty}$ preview tracking theory by state feedback for linear continuous-time Markovian jump systems $([11,15,16])$.

In this paper we study the stochastic optimal tracking problems with preview by state feedback for linear discrete-time Markovian jump systems on the finite time interval. We consider three different tracking problems according to the structure of preview information and give the control strategies for them respectively. The necessary and sufficient conditions for the solvability of our stochastic optimal tracking problem with preview are given by coupled Riccati difference equations with terminal conditions. Correspondingly compensators introducing future information are coupled with each other. It is our very important point in this paper. Finally we consider numerical examples and verify the effectiveness of our preview tracking theory.

\section{Problem Formulation}

Let $(\Omega, \mathcal{F}$, Prob $)$ be a probability space and, on this space, consider the following linear discrete-time timevarying system with reference signal and Markovian mode transitions.

$$
\begin{array}{r}
x(k+1)=A_{d, m(k)}(k) x(k)+G_{d, m(k)}(k) \omega_{d}(k) \\
+B_{2 d, m(k)}(k) u_{d}(k)+B_{3 d, m(k)}(k) r_{d}(k) \\
x(0)=x_{0}, m(0)=i_{0} \\
z_{d}(k)=C_{1 d, m(k)}(k) x(k)+D_{12 d, m(k)}(k) u_{d}(k) \\
+D_{13 d, m(k)}(k) r_{d}(k)
\end{array}
$$

where $x \in \mathbf{R}^{\mathrm{n}}$ is the state, $\omega_{d} \in \mathbf{R}^{\mathbf{P}_{\mathbf{d}}}$ is the exogenous random noise, $u_{d} \in \mathbf{R}^{\mathrm{m}}$ is the control input, $z_{d} \in \mathbf{R}^{\mathrm{k}_{\mathbf{d}}}$ 
is the controlled output, and $r_{d}(\cdot) \in \mathbf{R}^{\mathbf{r}_{\mathbf{d}}}$ is known or measurable reference signal. $x_{0}$ is an unknown initial state and $i_{0}$ is a given initial mode.

$\{m(k)\}$ is a Markov process taking values on the finite set $\phi=\left\{1,2, \cdots, N^{*}\right\}$ with the following transition probabilities:

$$
\operatorname{Prob}\{m(k+1)=j \mid m(k)=i\}:=p_{d, i j}(k)
$$

where $p_{d, i j}(k) \geq 0$ is also the transition rate at the jump instant from the state $i$ to $j, i \neq j$, and $\sum_{j=1}^{N^{*}} p_{d, i j}(k)=$ 1. Let $P_{d}(k)=\left[p_{d, i j}(k)\right]$ be the transition probability matrix. We assume that all these matrices are of compatible dimensions. Throughout this paper the dependence of the matrices on $k$ will be omitted for the sake of notation simplification.

For this system (1), we assume the following conditions.

A1: $D_{12 d, m(k)}(k)$ is of full column rank for all $k$.

$$
\begin{array}{ll}
\mathrm{A} 2: & D_{12 d, m(k)}^{\prime}(k) C_{1 d, m(k)}(k)=0, \\
& D_{12 d, m(k)}^{\prime}(k) D_{13 d, m(k)}(k)=0 \\
\mathrm{~A} 3: & \mathrm{E}\{x(0)\}=\mu_{0}, \mathrm{E}\left\{\omega_{d}(k)\right\}=0, \\
& \mathrm{E}\left\{\omega_{d}(k) \omega_{d}^{\prime}(k) \mathbf{1}_{\{m(k)=i\}}\right\}=\Xi_{i}(k), \\
& \mathrm{E}\left\{x(0) x^{\prime}(0) \mathbf{1}_{\left\{m(0)=i_{0}\right\}}\right\}=Q_{i_{0}}(0), \\
& \mathrm{E}\left\{\omega_{d}(0) x^{\prime}(0) \mathbf{1}_{\left\{m(0)=i_{0}\right\}}\right\}=0, \\
& \mathrm{E}\left\{\omega_{d}(k) x^{\prime}(k) \mathbf{1}_{\{m(k)=i\}}\right\}=0, \\
& \mathrm{E}\left\{\omega_{d}(k) u_{d}^{\prime}(k) \mathbf{1}_{\{m(k)=i\}}\right\}=0
\end{array}
$$

where the indicator function $\mathbf{1}_{\{m(k)=i\}}:=1$ if $m(k)=i$, and $\mathbf{1}_{\{m(k)=i\}}:=0$ if $m(k) \neq i$.

The optimal tracking problems we address in this section for the system (1) are to design control laws $u_{d}(\cdot) \in l_{2}[0, N-1]$ over the finite horizon $[0, N]$, using the information available on the known part of the reference signal $r_{d}(\cdot)$ and minimizing the sum of the energy of $z_{d}(\cdot)$, for the given initial mode $i_{0}$ and the given distribution of $x_{0}$. Considering the stochastic mode transitions and the average of the performance indices over the statistics of the unknown part of $r_{d}$, we define the following performance index.

$$
\begin{array}{r}
J_{d T}\left(x_{0}, u_{d}, r_{d}\right) \\
:=\sum_{k=0}^{N} \mathrm{E}_{\bar{R}_{k}}\left\{\left\|C_{1 d, m(k)} x(k)+D_{13 d, m(k)} r_{d}(k)\right\|^{2}\right\} \\
+\sum_{k=0}^{N-1} \mathrm{E}_{\bar{R}_{k}}\left\{\left\|D_{12 d, m(k)} u_{d}(k)\right\|^{2}\right\}
\end{array}
$$

$\mathrm{E}_{\bar{R}_{k}}$ means the expectation over $\bar{R}_{k+h}, h$ is the preview length of $r_{d}(k)$, and $\bar{R}_{k}$ denotes the future information on $r_{d}$ at the current time $k$, i.e., $\bar{R}_{k}:=\left\{r_{d}(l) ; k<l \leq\right.$ N\}.

We consider three different tracking problems according to the information structures (preview lengths) of $r_{d}$ as follows. i) Optimal Tracking of Causal $\left\{r_{d}(\cdot)\right\}$ :

In this case, $\left\{r_{d}(k)\right\}$ is measured on-line, i.e., at time $k, r_{d}(l)$ is known only for $l \leq k$.

ii) Optimal Fixed-Preview Tracking:

In this case, it is assumed that, at the current time $k$, $r_{d}(l)$ is known for $l \leq \min (N, k+h)$, where $h$ is the preview length.

iii) Optimal Tracking of Noncausal $\left\{r_{d}(\cdot)\right\}$ :

In this case, the signal $\left\{r_{d}(k)\right\}$ is assumed to be known a priori for the whole time interval $k \in[0, N]$.

In order to solve these three problems, we formulate the following optimization problem for the system (1) and the performance index (2).

The Stochastic LQ Optimal Tracking Problem by State Feedback:

Consider the system (1) and the performance index (2), and assume the conditions A1, A2 and A3. Then, find $\left\{u_{d}^{*}\right\}$ minimizing the performance index (2) where the control strategy $u_{d}^{*}(k), 0 \leq k \leq N-1$, is based on the information $R_{k+h}:=\left\{r_{d}(l) ; 0 \leq l \leq k+h\right\}$ with $0 \leq h \leq N$.

\section{Design of Tracking Controllers}

Now we consider the coupled Riccati difference equations $([4][5])$

$$
\begin{array}{r}
X_{i}(k)=A_{d, i}^{\prime} \mathcal{E}_{i}(X(k+1), k) A_{d, i}+C_{1 d, i}^{\prime} C_{1 d, i} \\
-F_{2, i}^{\prime} T_{2, i} F_{2, i}(k) k=0,1, \cdots
\end{array}
$$

where $\mathcal{E}_{i}(X(k+1), k)=\sum_{j=1}^{N^{*}} p_{d, i j}(k) X_{j}(k+1)$, $X(k)=\left(X_{1}(k), \cdots, X_{N^{*}}(k)\right)$

$$
\begin{aligned}
& T_{2, i}(k)=D_{12 d, i}^{\prime} D_{12 d, i}+B_{2 d, i}^{\prime} \mathcal{E}_{i}(X(k+1), k) B_{2 d, i}, \\
& R_{2, i}(k)=B_{2 d, i}^{\prime} \mathcal{E}_{i}(X(k+1), k) A_{d, i}, \\
& F_{2, i}(k)=-T_{2, i}^{-1} R_{2, i}(k) .
\end{aligned}
$$

We also consider the following scalar coupled difference equations.

$$
\begin{aligned}
\alpha_{i}(k)=\mathcal{E}_{i}( & \alpha(k+1), k) \\
& +\operatorname{tr}\left\{G_{d, i} \Xi_{i}(k) G_{d, i}^{\prime} \mathcal{E}_{i}(X(k+1), k)\right\}
\end{aligned}
$$

where $\mathcal{E}_{i}(\alpha(k+1), k)=\sum_{j=1}^{N^{*}} p_{d, i j}(k) \alpha_{j}(k+1)$ and $\alpha(k)=\left(\alpha_{1}(k), \cdots, \alpha_{N^{*}}(k)\right)$.

Remark 3.1 Note that these coupled Riccati difference equations and coupled scalar difference equations are the same as ones for the standard stochastic linear quadratic $(L Q)$ optimization problem of linear discretetime Markovian jump systems not considering any exogeneous reference signals nor any preview information $([4,5])$.

Remark 3.2 Also note that, using the terminal conditions $\alpha_{i}(N)=0$, we can solve the difference equations 
(4) backward as follows:

$$
\begin{array}{r}
\alpha_{i}(0)=\sum_{k=0}^{N-1} \operatorname{tr}\left\{G_{d, i} \Xi_{i}(k) G_{d, i}^{\prime} \mathcal{E}_{i}(X(k+1), k)\right\}, \\
1 \leq i \leq N^{*}
\end{array}
$$

In detail, refer to [5].

We obtain the following necessary and sufficient conditions for the solvability of the stochastic LQ optimal tracking problem and an optimal control strategy for it.

Proposition 3.1 Consider the system (1) and the performance index (2). Suppose A1, A2 and A3. Then the Stochastic LQ Optimal Tracking Problem by State Feedback for (1) and (2) is solvable if and only if there exist matrices $X_{i}(k)>O$ and scalar functions $\alpha_{i}(k), i=1, \cdots, N^{*}$ satisfying the conditions $X_{i}(N)=C_{1 d, i}^{\prime}(N) C_{1 d, i}(N)$ and $\alpha_{i}(N)=0$ such that the coupled Riccati equations (3) and the coupled scalar equations (4) hold over $[0, N]$. Moreover an optimal control strategy for our tracking problem (1) and (2) is given by

$$
\begin{array}{r}
u_{d}^{*}(k)=F_{2, i}(k) x(k \tau)+\mathbf{D}_{u, i}(k) r_{d}(k) \\
+\mathbf{D}_{\theta u, i} \mathcal{E}_{i}\left(\theta_{c}(k+1), k\right) \\
\quad \text { for } i=1, \cdots, N^{*}
\end{array}
$$

where

$$
\begin{gathered}
\mathbf{D}_{u, i}(k)=-T_{2, i}^{-1}(k) B_{2 d, i}^{\prime} \mathcal{E}_{i}(X(k+1), k) B_{3 d, i} \\
\text { and } \mathbf{D}_{\theta u, i}(k)=-T_{2, i}^{-1}(k) B_{2 d, i}^{\prime} . \\
\text { Let } \theta_{i}(k), i=1, \cdots, N^{*}, k \in[0, N] \text { satisfy } \\
\left\{\begin{array}{c}
\theta_{i}(k)=\bar{A}_{d, i}^{\prime}(k) \mathcal{E}_{i}(\theta(k+1), k)+\bar{B}_{d, i}(k) r_{d}(k) \\
\theta_{i}(N)=C_{1, i}^{\prime} D_{13, i} r_{d}(N)
\end{array}\right.
\end{gathered}
$$

where $\mathcal{E}_{i}(\theta(k+1), k)=\sum_{j=1}^{N^{*}} p_{d, i j}(k) \theta_{j}(k+1)$ and $\theta(k)=\left(\theta_{1}(k), \cdots, \theta_{N^{*}}(k)\right)$,

$$
\begin{aligned}
\bar{A}_{d, i}(k)=A_{d, i}- & \mathbf{D}_{\theta u, i}^{\prime} T_{2, i} F_{2, i}(k), \\
\bar{B}_{d, i}(k)=A_{d, i}^{\prime} \mathcal{E}_{i}(X(k+1), k) B_{3 d, i} & \quad-F_{2, i}^{\prime} T_{2, i} \mathbf{D}_{u, i}(k)+C_{1 d, i}^{\prime} D_{13 d, i},
\end{aligned}
$$

and $\theta_{c, i}(k)$ is the 'causal' part of $\theta_{i}(\cdot)$ at time $k$. This $\theta_{c, i}$ is the expected value of $\theta_{i}$ over $\bar{R}_{k}$ and given by

$$
\left\{\begin{array}{c}
\theta_{c, i}(l)=\bar{A}_{d, i}^{\prime}(l) \mathcal{E}_{i}\left(\theta_{c}(l+1), l\right)+\bar{B}_{d, i}(l) r_{d}(l) \\
k+1 \leq l \leq k+h \\
\theta_{c, i}(k+h+1)=0 \text { if } k+h \leq N-1 \\
\theta_{c, i}(k+h)=C_{1, i}^{\prime} D_{13, i} r_{d}(N) \text { if } k+h=N
\end{array}\right.
$$

where $\mathcal{E}_{i}\left(\theta_{c}(k+1), k\right)=\sum_{j=1}^{N^{*}} p_{d, i j}(k) \theta_{c, j}(k+1)$ and $\theta_{c}(k)=\left(\theta_{c, 1}(k), \cdots, \theta_{c, N^{*}}(k)\right)$. Moreover, the optimal value of the performance index is

$$
J_{d T}\left(x_{0}, u_{d}^{*}, r_{d}\right)
$$

$$
\begin{aligned}
& =\operatorname{tr}\left\{Q_{i_{0}} X_{i_{0}}\right\}+\alpha_{i_{0}}(0)+\mathbf{E}_{\bar{R}_{0}}\left\{2 \theta_{i_{0}}^{\prime} x_{0}\right\} \\
& +\sum_{k=0}^{N-1} \mathbf{E}_{\bar{R}_{k}}\left\{\| T_{2, m(k)}^{1 / 2} \mathbf{D}_{\theta u, m(k)}(k)\right. \\
& \left.\quad \times \mathcal{E}_{m(k)}\left(\theta_{c}^{-}(k+1), k\right) \|^{2}\right\}+\bar{J}_{d}\left(r_{d}\right)
\end{aligned}
$$

where $\theta_{c, m(k)}^{-}(k)=\theta_{m(k)}(k)-\theta_{c, m(k)}(k), k \in[0, N]$, $\mathcal{E}_{i}\left(\theta_{c}^{-}(k+1), k\right)=\sum_{j=1}^{N^{*}} p_{d, i j}(k) \theta_{c, j}^{-}(k+1)$ and $\theta_{c}^{-}(k)=\left(\theta_{c, 1}^{-}(k), \cdots, \theta_{c, N^{*}}^{-}(k)\right)$,

$$
\begin{gathered}
\bar{J}_{d}\left(r_{d}\right)=\mathbf{E}_{\bar{R}_{N}}\left\{\|\left. D_{13 d, m(N)} r_{d}(N)\right|^{2}\right\} \\
+\sum_{k=0}^{N-1} \mathbf{E}_{\bar{R}_{k}}\left\{-\left\|T_{2, m(k)}^{1 / 2} \mathbf{D}_{\theta u, m(k)}(k) \mathcal{E}_{m(k)}(\theta(k+1), k)\right\|^{2}\right. \\
-2 \mathcal{E}_{m(k)}\left(\theta^{\prime}(k+1), k\right) \mathbf{D}_{\theta u, m(k)}^{\prime} \\
\quad \times T_{2, m(k)} \mathbf{D}_{u, m(k)}(k) r_{d}(k) \\
+2 \mathcal{E}_{m(k)}\left(\theta^{\prime}(k+1), k\right) B_{3 d, m(k)} r_{d}(k) \\
\left.+J_{d, k, m(k)}\left(r_{d}\right)\right\}, \\
J_{d, k, m(k)}\left(r_{d}\right)=r_{d}^{\prime}(k)\left[-\mathbf{D}_{u, m(k)}^{\prime} T_{2, m(k)} \mathbf{D}_{u, m(k)}(k)\right. \\
+B_{3 d, m(k)}^{\prime} \mathcal{E}_{m(k)}(X(k+1), k) B_{3 d, m(k)} \\
\left.\quad+D_{13 d, m(k)}^{\prime} D_{13 d, m(k)}\right] r_{d}(k) .
\end{gathered}
$$

Remark 3.3 Note that each dynamics (6) of $\theta_{c, i}$ which composes the compensator introducing the preview information, is coupled on the other. It corresponds to the characteristic that the Riccati difference equations (3) are coupled with each other, which give the necessary and sufficient conditions for the solvability of the stochastic $L Q$ optimal tracking problem.

Remark 3.4 When the mode transition probabilities $p_{d, i j}(k)$ with $\sum_{j=1}^{N^{*}} p_{d, i j}(k)=1$ are known, we can easily calculate the probabilities $\pi_{i}(k)=\operatorname{Prob}\{m(k)=i\}$. These $\pi_{i}(k)$ are rather important in the case of output feedback problems. Moreover, in the case that the initial mode is random variable with some distributions, the optimal value of the performance index can be expressed using the backwrad solutions $\alpha_{i}(0)$ obtained in the it Remark 3.2 as follows:

$$
\begin{aligned}
& J_{d T}\left(x_{0}, u_{d}^{*}, r_{d}\right) \\
& =\sum_{i=1}^{N^{*}}\left[\operatorname{tr}\left\{\pi_{i}(0) Q_{i}(0) X_{i}(0)\right\}+\left\{\pi_{i}(0) 2 \theta_{i}(0)^{\prime} \mu_{0}\right\}\right. \\
& \quad+\sum_{k=0}^{N-1} \pi_{i}(k) \operatorname{tr}\left\{G_{d, i} \Xi_{i}(k) G_{d, i}^{\prime} \mathcal{E}_{i}(X(k+1), k)\right\} \\
& +\sum_{k=0}^{N-1} \pi_{i}(k)\left\{\| T_{2, i}^{1 / 2} \mathbf{D}_{\theta u, i}(k)\right. \\
& \left.\left.\quad \times \mathcal{E}_{i}\left(\theta_{c}^{-}(k+1), k\right) \|^{2}\right\}\right]+\bar{J}_{d}\left(r_{d}\right)
\end{aligned}
$$

where $\mathrm{E}\left\{x(0) x^{\prime}(0) \mathbf{1}_{\{m(0)=i\}}\right\}=Q_{i}(0)$ and we have used the fact that the probabilities $\pi_{i}(\cdot)$ are independent of the information $\bar{R}_{k+h}$. 


\section{(Proof)}

Sufficiency: Let $X_{i}(k)>O$ and $\alpha_{i}(k)$ be solutions to (3) and (4) over $[0, N]$ such that $X_{i}(N)=$ $C_{1 d, i}^{\prime}(N) C_{1 d, i}(N)$ and $\alpha_{i}(N)=0$. Define

$$
\begin{aligned}
& \phi_{k, m(k)} \\
& :=\mathrm{E}\left\{x^{\prime}(k+1) X_{m(k+1)}(k+1) x(k+1)\right. \\
& \left.+\alpha_{m(k+1)}(k+1) \mid x(k), m(k)\right\} \\
& -\left\{x^{\prime}(k) X_{m(k)}(k) x(k)+\alpha_{m(k)}(k)\right\}
\end{aligned}
$$

We first consider the case of $r_{d}(\cdot) \equiv 0$. Then the following equalities hold by the assumptions A3.

$$
\begin{gathered}
\mathbf{E}\left\{x^{\prime}(k+1) X_{m(k+1)}(k+1) x(k+1)\right. \\
\left.+\alpha_{m(k+1)}(k+1) \mid x(k), m(k)\right\} \\
=\mathrm{E}\left\{\left(A_{d, m(k)}(k) x(k)\right.\right. \\
\left.+G_{d, m(k)}(k) w_{d}(k)+B_{2 d, m(k)}(k) u_{d}(k)\right)^{\prime} \\
\times X_{m(k+1)}(k+1)\left(A_{d, m(k)}(k) x(k)\right. \\
\left.+G_{d, m(k)}(k) w_{d}(k)+B_{2 d, m(k)}(k) u_{d}(k)\right) \\
\left.+\alpha_{m(k+1)}(k+1) \mid x(k), m(k)\right\} \\
=\left(A_{d, m(k)}(k) x(k)+B_{2 d, m(k)}(k) u_{d}(k)\right)^{\prime} \\
\times \mathcal{E}_{m(k)}(X(k+1), k) \\
\quad \times\left(A_{d, m(k)}(k) x(k)+B_{2 d, m(k)}(k) u_{d}(k)\right) \\
+\operatorname{tr}\left\{G_{d, m(k)}(k) \Xi_{i}(k) G_{d, m(k)}^{\prime}(k) \mathcal{E}_{m(k)}(X(k+1), k)\right\} \\
\quad+\mathcal{E}_{m(k)}(\alpha(k+1), k)
\end{gathered}
$$

It can be shown that the following equality holds, using the system (1) and the coupled Riccati equations (3) and the coupled scalar equations (4).(cf. $[4,5]$ )

$$
\begin{aligned}
& \mathrm{E}_{\bar{R}_{k}}\left\{\phi_{k, m(k)}\right\}=\mathrm{E}_{\bar{R}_{k}}\left\{-\left\|z_{d}(k)\right\|^{2}\right. \\
& \left.\quad+\left\|T_{2, m(k)}^{1 / 2}(k)\left[u_{d}(k)-F_{2, m(k)}(k) x(k)\right]\right\|^{2}\right\}
\end{aligned}
$$

Moreover, in the genaral case that $\left\{r_{d}(\cdot)\right\}$ is arbitrary, we have the following equality.

$$
\begin{aligned}
& \mathrm{E}_{\bar{R}_{k}}\left\{\phi_{k, m(k)}\right\}=\mathrm{E}_{\bar{R}_{k}}\left\{-\left\|z_{d}(k)\right\|^{2}\right. \\
& +\| T_{2, m(k)}^{1 / 2}(k)\left[u_{d}(k)-F_{2, m(k)}(k) x(k)\right. \\
& \left.\quad-\mathbf{D}_{u, m(k)}(k) r_{d}(k)\right] \|^{2} \\
& \left.+2 x^{\prime}(k \tau) \bar{B}_{d, m(k)}(k) r_{d}(k)+J_{d, k, m(k)}\left(r_{d}\right)\right\}
\end{aligned}
$$

Notice that, in the right hand side of this equality, $J_{d, k, m(k)}\left(r_{d}\right)$, which means the tracking error without considering the effect of the preview information, is added. Now introducing the vector $\theta_{m(k)}$, which can include some preview information of the tracking signals,

$$
\begin{aligned}
& \mathrm{E}\left\{\theta_{m(k+1)}^{\prime}(k+1) x(k+1) \mid x(k), m(k)\right\} \\
& -\theta_{m(k)}^{\prime}(k) x(k) \\
& =\mathcal{E}_{m(k)}\left(\theta^{\prime}(k+1), k\right)\left(A_{d, m(k)} x(k)\right. \\
& \quad+G_{d, m(k)} \omega_{d}(k)+B_{2 d, m(k)} u_{d}(k) \\
& \left.\quad+B_{3 d, m(k)} r_{d}(k)\right)-\theta_{m(k)}^{\prime}(k) x(k)
\end{aligned}
$$

Then we obtain

$$
\begin{gathered}
\mathbf{E}_{\bar{R}_{k}}\left\{\phi_{k, m(k)}\right. \\
+2\left\{\mathbf{E}\left\{\theta_{m(k+1)}^{\prime}(k+1) x(k+1) \mid x(k), m(k)\right\}\right. \\
\left.\left.-\theta_{m(k)}^{\prime}(k) x(k)\right\}\right\} \\
=\mathbf{E}_{\bar{R}_{k}}\left\{-\left\|z_{d}(k)\right\|^{2}\right. \\
+\| T_{2, m(k)}^{1 / 2}(k)\left[u_{d}(k)-F_{2, m(k)}(k) x(k)\right. \\
\left.-\mathbf{D}_{u, m(k)}(k) r_{d}(k)\right] \|^{2} \\
+2 x^{\prime}(k \tau) \bar{B}_{d, m(k)}(k) r_{d}(k)+J_{d, k, m(k)}\left(r_{d}\right) \\
+2 \mathcal{E}_{m(k)}\left(\theta^{\prime}(k+1), k\right)\left(A_{d, m(k)} x(k)\right. \\
+G_{d, m(k)} \omega_{d}(k)+B_{2 d, m(k)} u_{d}(k) \\
\left.\left.+B_{3 d, m(k)} r_{d}(k)\right)-2 \theta_{m(k)}^{\prime}(k) x(k)\right\} \\
=\mathbf{E}_{\bar{R}_{k}}\left\{-\left\|z_{d}(k)\right\|^{2} \quad-\mathbf{D}_{u, m(k)}(k) r_{d}(k)\right. \\
+\| T_{2, m(k)}^{1 / 2}(k)\left[u_{d}(k)-F_{2, m(k)}(k) x(k)\right. \\
\left.-\mathbf{D}_{\theta u, m(k)}(k) \mathcal{E}_{m(k)}(\theta(k+1), k)\right] \|^{2} \\
\left.+\bar{J}_{d, k, m(k)}\left(r_{d}\right)\right\}
\end{gathered}
$$

where we have used the dynamics

$$
\theta_{i}(k)=\bar{A}_{d, i}^{\prime}(k) \mathcal{E}_{i}(\theta(k+1), k)+\bar{B}_{d, i}(k) r_{d}(k)
$$

to get rid of the terms that mix $r_{d}$ and $x$, or $\theta_{m(k)}$ and $x$. $\bar{J}_{d, k, m(k)}\left(r_{d}\right)$ means the tracking error including the preview information vector $\theta$ and can be expressed by

$$
\begin{gathered}
\bar{J}_{d, k, m(k)}\left(r_{d}\right) \\
=-\left\|T_{2, m(k)}^{1 / 2} \mathbf{D}_{\theta u, m(k)}(k) \mathcal{E}_{m(k)}(\theta(k+1), k)\right\|^{2} \\
-2 \mathcal{E}_{m(k)}\left(\theta^{\prime}(k+1), k\right) \mathbf{D}_{\theta u, m(k)}^{\prime} \\
\times T_{2, m(k)} \mathbf{D}_{u, m(k)}(k) r_{d}(k) \\
+2 \mathcal{E}_{m(k)}\left(\theta^{\prime}(k+1), k\right) B_{3 d, m(k)} r_{d}(k) \\
\quad+J_{d, k, m(k)}\left(r_{d}\right) .
\end{gathered}
$$

Taking the sum of the quantities (8) from $k=0$ to $k=N-1$ and adding $\left\|C_{1, m(k)} x(N)+D_{13, m(k)} r_{d}(N)\right\|^{2}$,

$$
\begin{aligned}
& \sum_{k=0}^{N-1} \mathbf{E}_{\bar{R}_{k}}\left\{\left\|z_{d}(k)\right\|^{2}\right\} \\
& +\left\|C_{1, m(k)} x(N)+D_{13, m(k)} r_{d}(N)\right\|^{2} \\
& +\sum_{k=0}^{N-1} \mathbf{E}_{\bar{R}_{k}}\left\{\phi_{k, m(k)}\right. \\
& +2\left\{\mathbf{E}\left\{\theta_{m(k+1)}^{\prime}(k+1) x(k+1) \mid x(k), m(k)\right\}\right. \\
& \left.=\sum_{k=0}^{\prime} \mathbf{E}_{\bar{R}_{k}(k)}\left\{\| \hat{u}_{d}(k) x(k)\right\}\right\} \\
& \quad-\mathbf{D}_{\theta u, m(k)}(k) \mathcal{E}_{m(k)}(\theta(k+1), k) \|_{T_{2, m(k)}}^{2}(k) \\
& +\left\|C_{1, m(k)} x(N)+D_{13, m(k)} r_{d}(N)\right\|^{2} \\
& \quad+\sum_{k=0}^{N-1} \mathbf{E}_{\bar{R}_{k}}\left\{\bar{J}_{d, k, m(k)}\left(r_{d}\right)\right\}
\end{aligned}
$$


where

$$
\hat{u}_{d}(k)=u_{d}(k)-F_{2, m(k)}(k) x(k)-\mathbf{D}_{u, m(k)}(k) r_{d}(k) .
$$

Since the left hand side reduces to

$$
\begin{aligned}
& \sum_{k=0}^{N-1} \mathrm{E}_{\bar{R}_{k}}\left\{\left\|z_{d}(k)\right\|^{2}\right\} \\
& \quad+\left\|C_{1, m(k)} x(N)+D_{13, m(k)} r_{d}(N)\right\|^{2} \\
& +2 \theta_{m(N)}^{\prime}(N) x(N)+x^{\prime}(N) X_{m(N)}(N) x(N) \\
& \quad+\alpha_{m(N)}(N) \\
& \quad+\mathbf{E}_{\bar{R}_{0}}\left\{-2 \theta_{i_{0}}^{\prime}(0) x(0)-x_{0}^{\prime} X_{i_{0}}(0) x_{0}-\alpha_{i_{0}}(0)\right\}
\end{aligned}
$$

we obtain

$$
\begin{aligned}
& \quad J_{d T}\left(x_{0}, u_{d}, r_{d}\right) \\
& =\operatorname{tr}\left\{Q_{i_{0}} X_{i_{0}}\right\}+\alpha_{i_{0}}(0)+\mathbf{E}_{\bar{R}_{0}}\left\{2 \theta_{i_{0}}^{\prime} x_{0}\right\} \\
& +\sum_{k=0}^{N-1} \mathbf{E}_{\bar{R}_{k}}\left\{\| \hat{u}_{d}(k)\right. \\
& \left.\quad-\mathbf{D}_{\theta u, m(k)}(k) \mathcal{E}_{m(k)}(\theta(k+1), k) \|_{T_{2, m(k)}(k)}^{2}\right\} \\
& \quad+\bar{J}_{d}\left(r_{d}\right)
\end{aligned}
$$

where we have used the terminal conditions $X_{i}(N)=$ $C_{1 d, i}^{\prime}(N) C_{1 d, i}(N), \theta_{i}(N)=C_{1 d, i}^{\prime}(N) D_{13 d, i}(N) r_{d}(N)$ and $\alpha_{i}(N)=0$. Note that $\bar{J}_{d}\left(r_{d}\right)$ is independent of $u_{d}$ and $x_{0}$. Since the average of $\theta_{c, m(k)}^{-}$over $\bar{R}_{k}$ is zero, including the 'causal' part $\theta_{c, m(k)}(k)$ of $\theta(\cdot)$ at time $k$, we adopt

$$
\hat{u}_{d}^{*}(k)=\mathbf{D}_{\theta u, m(k)}(k) \mathcal{E}_{m(k)}\left(\theta_{c}(k+1), k\right)
$$

as the minimizing control strategy. Then finally we obtain

$$
\begin{aligned}
& J_{d T}\left(x_{0}, u_{d}, r_{d}\right) \\
& =\operatorname{tr}\left\{Q_{i_{0}} X_{i_{0}}\right\}+\alpha_{i_{0}}(0)+\mathbb{E}_{\bar{R}_{0}}\left\{2 \theta_{i_{0}}^{\prime} x_{0}\right\} \\
& +\sum_{k=0}^{N-1} \mathrm{E}_{\bar{R}_{k}}\left\{\| \hat{u}_{d}(k)\right. \\
& \left.-\mathbf{D}_{\theta u, m(k)}(k) \mathcal{E}_{m(k)}(\theta(k+1), k) \|_{T_{2, m(k)}(k)}^{2}\right\} \\
& +\bar{J}_{d}\left(r_{d}\right) \\
& \geq \operatorname{tr}\left\{Q_{i_{0}} X_{i_{0}}\right\}+\alpha_{i_{0}}(0)+\mathrm{E}_{\bar{R}_{0}}\left\{2 \theta_{i_{0}}^{\prime} x_{0}\right\} \\
& +\sum_{k=0}^{N-1} \mathrm{E}_{\bar{R}_{k}}\left\{\left\|\mathbf{D}_{\theta u, m(k)}(k) \mathcal{E}_{m(k)}\left(\theta^{-}(k+1), k\right)\right\|_{T_{2, m(k)}(k)}^{2}\right\} \\
& +\bar{J}_{d}\left(r_{d}\right) \\
& =J_{d T}\left(x_{0}, u_{d}^{*}, r_{d}\right) \text {. }
\end{aligned}
$$

which concludes the proof of sufficiency.

Necessity: Because of arbitrariness of the reference signal $r_{d}(\cdot)$, by considering the case of $r_{d}(\cdot) \equiv 0$, one can easily deduce the necessity for the solvability of our stochastic LQ optimal tracking problem. ([4, 5]) (QED)

Next, according to Proposition 3.1, we present solution to each of the three optimal tracking problems by state feedback.
Theorem 3.1 Consider the system (1) and the performance index (2). Suppose A1, A.2 and A.3. Then each of the Optimal tracking problems for (1) and (2) is solvable by state feedback if and only if there exist matrices $X_{i}(k)>O$ and scalar functions $\alpha_{i}(k), i=1, \cdots, N^{*}$, satisfying the conditions $X_{i}(N)=$ $C_{1 d, i}^{\prime}(N) C_{1 d, i}(N)$ and $\alpha_{i}(N)=0$ such that the coupled Riccati equations (3) and the coupled scalar equations (4) hold over $[0, N]$. Moreover, the following results hold using

$$
\begin{aligned}
& K_{d, x, i}(k)=F_{2, i}(k), K_{r_{d}, i}(k)=\mathbf{D}_{u, i}(k) \\
& \quad \text { and } K_{d, \theta, i}(k)=\mathbf{D}_{\theta u, i}(k) .
\end{aligned}
$$

i) The control law for the Optimal Tracking of Causal $\mathrm{r}_{\mathrm{d}}(\cdot)$ is

$$
u_{d, s 1}(k)=K_{d, x, i}(k) x(k)+K_{r_{d}, i}(k) r_{d}(k)
$$

and

$$
\begin{aligned}
& J_{d T}\left(x_{0}, u_{d, s 1}, r_{d}\right) \\
& =\operatorname{tr}\left\{Q_{i_{0}} X_{i_{0}}\right\}+\alpha_{i_{0}}(0)+\mathbf{E}_{\bar{R}_{0}}\left\{2 \theta_{i_{0}}^{\prime} x_{0}\right\} \\
& +\sum_{k=0}^{N-1} \mathbf{E}_{\bar{R}_{k}}\left\{\| T_{2, m(k)}^{1 / 2} \mathbf{D}_{\theta u, m(k)}(k)\right. \\
& \left.\quad \times \mathcal{E}_{m(k)}(\theta(k+1), k) \|^{2}\right\}+\bar{J}_{d}\left(r_{d}\right)
\end{aligned}
$$

ii) The control law for the Optimal Fixed-Preview Tracking is

$$
\begin{array}{r}
u_{d, s 2}(k)=K_{d, x, i}(k) x(k)+K_{r_{d}, i}(k) r_{d}(k) \\
+K_{d, \theta, i}(k) \mathcal{E}_{i}\left(\theta_{c}(k+1), k\right)
\end{array}
$$

with $\theta_{c, i}(\cdot)$ given by $(6)$. Moreover, $J_{T}\left(x_{0}, u_{d, s 2}, r_{d}\right)$ coincides with (7) . iii) The control law for the Optimal Tracking of Noncausal $\mathrm{r}_{\mathrm{d}}(\cdot)$ is

$$
\begin{aligned}
u_{d, s 3}=K_{d, x, i}(k) x(k) & +K_{r_{d}, i}(k) r_{d}(k) \\
& +K_{d, \theta, i}(k) \mathcal{E}_{i}(\theta(k+1), k)
\end{aligned}
$$

with $\theta_{i}(\cdot)$ given by (5) and

$$
\begin{aligned}
& J_{d T}\left(x_{0}, u_{d, s 3}, r_{d}\right) \\
= & \operatorname{tr}\left\{Q_{i_{0}} X_{i_{0}}\right\}+\alpha_{i_{0}}(0)+\mathbf{E}_{\bar{R}_{0}}\left\{2 \theta_{i_{0}}^{\prime} x_{0}\right\}+\bar{J}_{d}\left(r_{d}\right)
\end{aligned}
$$

since $\theta_{i}(k)=\theta_{c, i}(k), \forall k \in[0, N]$.

\section{Numerical Examples}

In this section, we study numerical examples to demonstrate the effectiveness of the stochastic LQ optimal tracking design theory presented in the previous section.

We consider the following two mode systems and assume that the system parameters are as follows: 
$(\mathrm{cf} \cdot[2,17])$

$$
\begin{array}{r}
x(k+1)=A_{d, m(k)}(k) x(k)+G_{d}(k) \omega_{d}(k) \\
+B_{2 d}(k) u_{d}(k)+B_{3 d, m(k)}(k) r_{d}(k) \\
x(0)=x_{0}, m(0)=i_{0},\{m(k)\}=\{1,2\} \\
z_{d}(k)=C_{1 d, m(k)}(k) x(k)+D_{12 d}(k) u_{d}(k) \\
+D_{13 d}(k) r_{d}(k)
\end{array}
$$

- Mode 1 :

$$
\begin{aligned}
& A_{d, 1}=\left[\begin{array}{cc}
0 & 1 \\
-0.8 & 1.6
\end{array}\right], \quad A_{d, 2}=\left[\begin{array}{cc}
0 & 1 \\
1.6 & -0.2
\end{array}\right], \\
& B_{2 d}=\left[\begin{array}{l}
0 \\
1
\end{array}\right], B_{3 d, 1}=\left[\begin{array}{c}
1.5 \\
0
\end{array}\right], B_{3 d, 1}=\left[\begin{array}{c}
1.8 \\
0
\end{array}\right],
\end{aligned}
$$

and

$$
\begin{aligned}
& C_{1 d, 1}=\left[\begin{array}{cc}
-0.5 & 0.2 \\
0 & 0
\end{array}\right], C_{1 d, 2}=\left[\begin{array}{cc}
-0.5 & 0.1 \\
0 & 0
\end{array}\right], \\
& G_{d}=\left[\begin{array}{c}
0 \\
0.1
\end{array}\right], D_{12 d}=\left[\begin{array}{c}
0 \\
0.1
\end{array}\right], D_{13 d}=\left[\begin{array}{c}
-1.0 \\
0
\end{array}\right]
\end{aligned}
$$

It is assumed that $r_{d}(\cdot)$ is not always a priori known over the whole time interval. Let

$$
P_{d}=\left[\begin{array}{ll}
0.3 & 0.7 \\
0.6 & 0.4
\end{array}\right]
$$

be a stationary transition matrix of $\{m(k)\}$. We set $x_{0}=\operatorname{col}(0,0)$ and $i_{0}=1$.

Then we introduce the following objective function.

$$
\begin{array}{r}
J_{d T}\left(x_{0}, u_{d}, r_{d}\right) \\
=\sum_{k=0}^{N} \mathrm{E}_{\bar{R}_{k}}\left\{\left\|C_{1 d, m(k)} x(k)+D_{13 d, m(k)} r_{d}(k)\right\|^{2}\right\} \\
+\sum_{k=0}^{N-1} \mathrm{E}_{\bar{R}_{k}}\left\{\left\|D_{12 d, m(k)} u_{d}(k)\right\|^{2}\right\}
\end{array}
$$

By the term $B_{3 d, i} r_{d}(k), i=1,2$, the tracking performance can be expected to be improved as [2], [17] and so on. $N$ is assumed to be very large. The paths of $m(k)$ are generated randomly, and the performances are compared under the same condition, that is, the same set of the paths so that the performances can be easily compared.

First we consider the system (9) with only mode 1 and no mode transition over the time interval $k \in[0,100]$. For this system (9) with mode 1, we apply the results of our stochastic LQ tracking design theory for $r_{d}(k)=$ $0.5 \sin (\pi k / 20)$ and $r_{d}(k)=0.5 \sin (\pi k / 100)$ with various step lengths of preview, and show the simulation results. It is shown in Fig. 1 for $r_{d}(k)=0.5 \sin (\pi k / 20)$ and Fig. 2 for $r_{d}(k)=0.5 \sin (\pi k / 100)$ that increasing the preview steps from $h=0$ to $h=1,2,3,4$ improves the tracking performance. In fact, the square

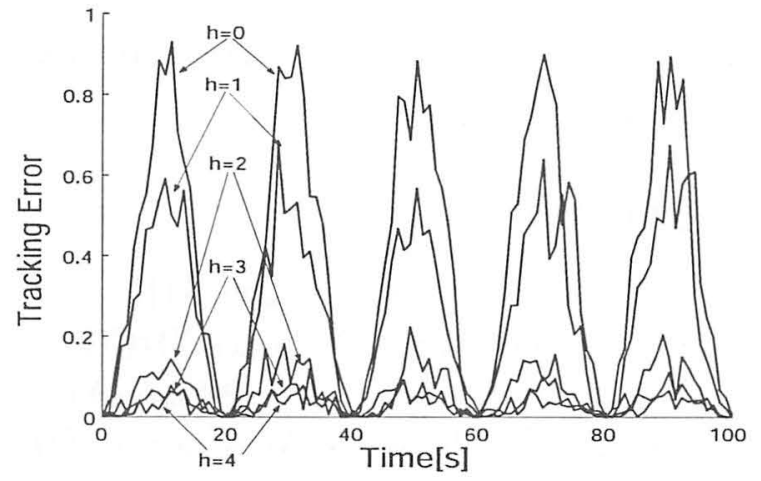

Fig. 1: Mode 1: The errors of tracking $r_{d}(k)=$ $0.5 \sin (\pi k / 20)$ for various preview lengths

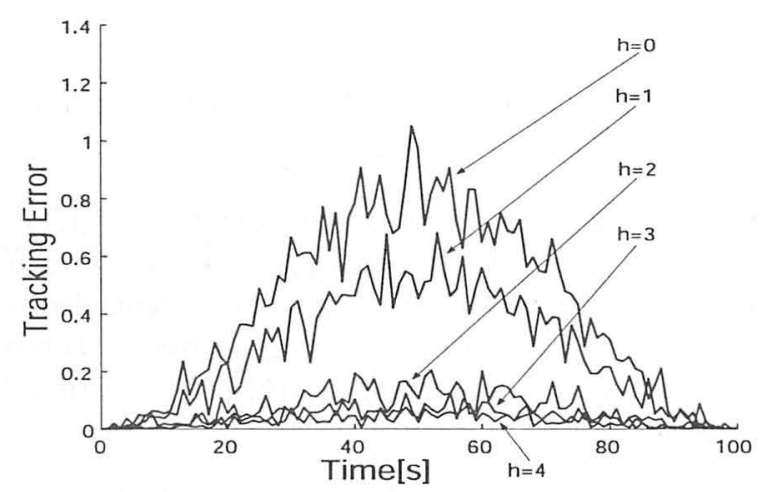

Fig. 2: Mode 1: The errors of tracking $r_{d}(k)=$ $0.5 \sin (\pi k / 100)$ for various preview lengths

values $\left\|C_{1 d, 1} x(k)+D_{13 d} r_{d}(k)\right\|^{2}$ of the tracking errors are shown in Fig. $\mathbf{1}$ and Fig. 2 and it is clear the tracking error decreases as increasing the preview steps by these figures.

Next we consider the system (9) with only mode 2 and no mode transition over the time interval $k \in[0,100]$. For this system (9) with mode 2, we apply the results of our stochastic optimal tracking theory for $r_{d}(k)=$ $0.5 \sin (\pi k / 20)$ and $r_{d}(k)=0.5 \sin (\pi k / 100)$ with various step lengths of preview, and show the simulation results. It is shown in Fig. 3 for $r_{d}(k)=0.5 \sin (\pi k / 20)$ and Fig. 4 for $r_{d}(k)=0.5 \sin (\pi k / 100)$ that the tracking error decreases as increasing the preview steps from $h=0$ to $h=1,2,3,4$ and the tracking performance is improved.

From these simulation reselts, we can expect that increasing the preview steps improves the tracking performance for the whole system considering the mode transition rate $P_{d}$. Now we consider the whole system with the mode transition rate $P_{d}$. For this system, we apply the results of the our stochastic optimal tracking theory for $r_{d}(k)=0.5 \sin (\pi k / 20)$ and $r_{d}(k)=0.5 \sin (\pi k / 100)$ 


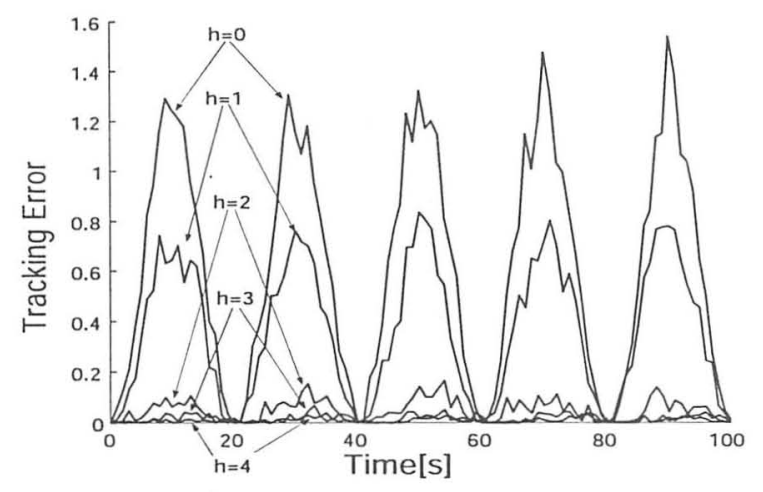

Fig. 3: Mode 2: The errors of tracking $r_{d}(k)=$ $0.5 \sin (\pi k / 20)$ for various preview lengths

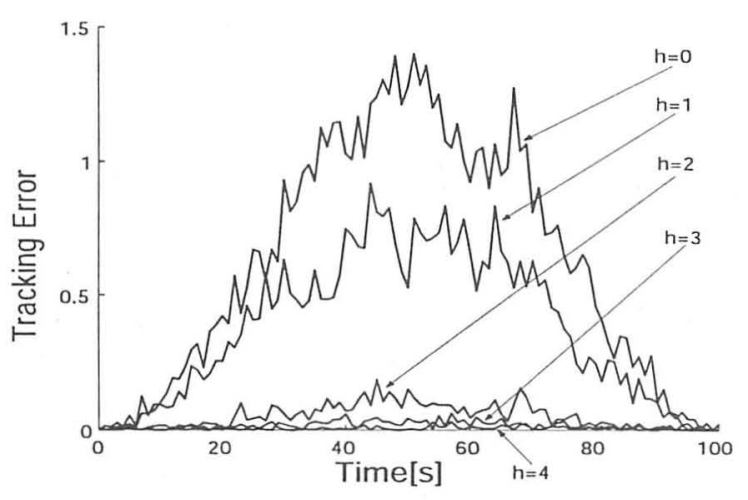

Fig. 4: Mode 2: The errors of tracking $r_{d}(k)=$ $0.5 \sin (\pi k / 100)$ for various preview lengths

with various step lengths of preview, and show the simulation results. It is shown that increasing the preview steps from $h=0$ to $h=1,2,3,4$, improves the tracking performance in Fig. 5 for $r_{d}(k)=0.5 \sin (\pi k / 20)$ and Fig. 6 for $r_{d}(k)=0.5 \sin (\pi k / 100)$. Finally the tracking responses of the whole system for $r_{d}(k)=$ $0.5 \sin (\pi k / 100)$ with various preview lengths are shown in Fig. 7. It is clear that, in the noncausal tracking case, better tracking performance can be obtained for the reference signal which does not include any periodic signals over the whole time interval.

\section{Concluding Remarks}

In this paper we have presented the stochastic linear quadratic (LQ) optimal tracking control theory considering the preview information by state feedback for the linear discrete-time Markovian jump systems, which are a class of stochastic switching systems, and verified the effectiveness of our design theory by numerical examples. The compensators introducing the preview information of the reference signals are coupled with each

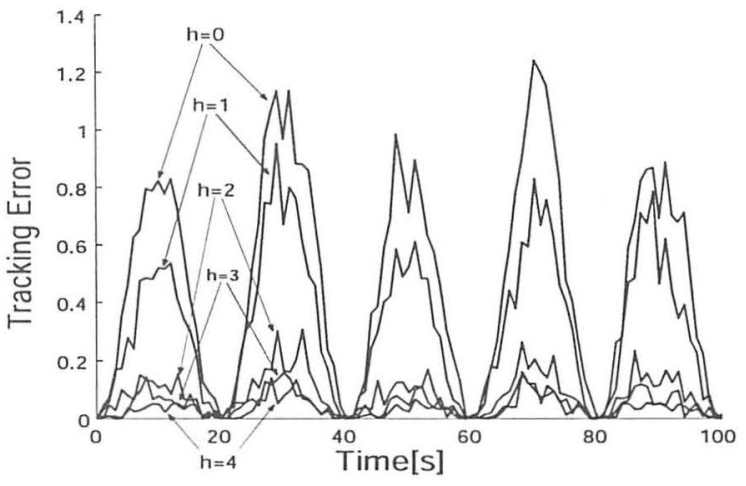

Fig. 5: The whole system consisting of mode 1 and mode 2: The errors of tracking $r_{d}(k)=0.5 \sin (\pi k / 20)$ for various preview lengths

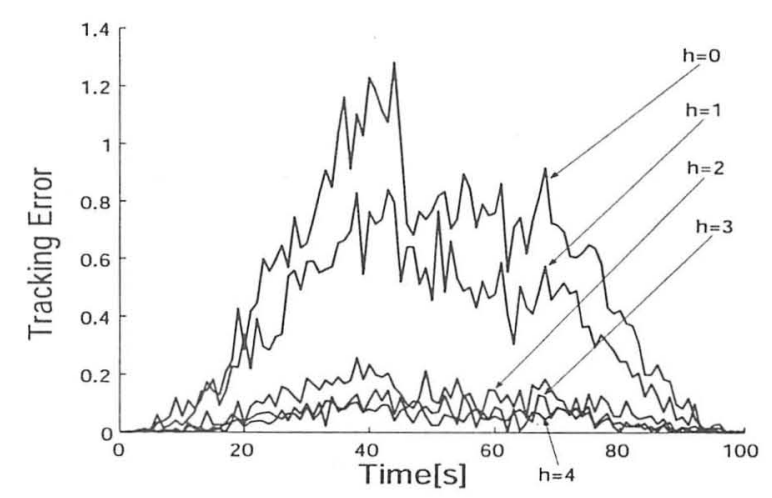

Fig. 6: The whole system consisting of mode 1 and mode 2: The errors of tracking $r_{d}(k)=0.5 \sin (\pi k / 100)$ for various preview lengths

other. The state feedback theory presented in this paper can be extended to output feedback theory. In order to construct the output feedback theory, we need design some LMMSE (linear minimum mean square error) filters adapted to effects of preview feedforward compensation. The partial observation problems for Markovian jump systems are classified into the case that the transition modes are known and the one that they cannot be observed. These design theory will be reported elsewhere. With regard to perfectly noncausal optimal tracking theory for general linear switched systems, refer to $([12])$.

\section{References}

[1] E. K. Boukas: Stochastic Switching Systems: Analysis and Design, Birkhauser, Boston, 2006.

[2] A. Cohen and U. Shaked: Linear Discrete-Time $\mathrm{H}_{\infty}$-Optimal Tracking with Preview, IEEE Trans. 


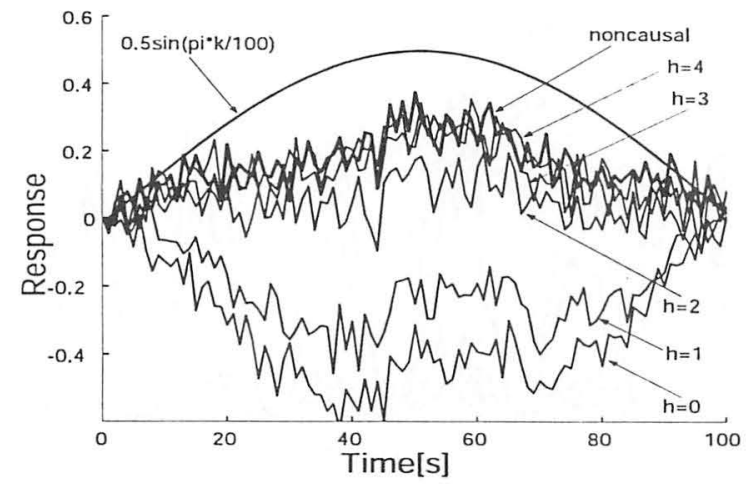

Fig. 7: The whole system consisting of mode 1 and mode 2: The responses for $r_{d}(k)=0.5 \sin (\pi k / 100)$ and various preview lengths

Automat. Contr., Vol. 42, pp.270-276, 1997.

[3] O. L. V. Costa and E. F. Tuesta: Finite Horizon Quadratic Optimal Control and a Separation Principle for Markovian Jump Linear Systems, IEEE Trans. Automat. Contr., Vol. 48, No. 10, pp. 18361842, 2003.

[4] O. L. V. Costa, M. D. Fragoso and R. P. Marques: Discrete-Time Markov Jump Linear Systems, Springer, London, 2005.

[5] M. D. Fragoso: Discrete-Time Jump LQG Problem, Int. J. Systems Science, Vo. 20, No. 12 pp. 2539-2545, 1989.

[6] M. D. Fragoso, J. B. R. do Val and D. L. Pinto Junior: Jump Linear $\mathrm{H}_{\infty}$ Control: the discretetime case, Control-Theory and Advanced Technology, Vol. 10, No. 4, pp. 1459-1474, 1995.

[7] E. Gershon, D. J. N. Limebeer, U. Shaked and I. Yaesh: Stochastic $\mathrm{H}_{\infty}$ Tracking with Preview for State-Multiplicative Systems, IEEE Trans. Automat. Contr., Vol. 49, pp.2061-2068, 2004.

[8] E. Gershon, U. Shaked and I. Yaesh: $\mathrm{H}_{\infty}$ tracking of linear continuous-time systems with stochastic uncertainties and preview, Int. J. Robust and Nonlinear Control, Vol. 14, pp. 607-626, 2004.

[9] H. Katayama and A. Ichikawa: $\mathrm{H}_{\infty}$-Control with State Feedback for Time-Varying Discrete Systems, Int. J. Contr., Vol. 60, No. 3, pp. 451-465, 1994.

[10] M. Mariton: Jump Linear Systems in Automatic Control, Marcel Dekker, New York, 1990.

[11] G. Nakura: Optimal Tracking with Preview for Linear Continuous-Time Markovian Jump Systems, Proceedings of The 42th Workshop on Dis- crete Event Systems, pp.45-50, Toyonaka, Osaka, Japan, 2007.

[12] G. Nakura: Noncausal Optimal Tracking for Linear Switched Systems; Proceedings of The 11th International Workshop of Hybrid Systems : Computation and Control (HSCC2008), St. Louis, MO, USA (2008, 4, 22-24), Springer LNCS Vol. 4981, pp. $372-385,2008$.

[13] G. Nakura: $\mathrm{H}_{\infty}$ Tracking with Preview for Linear Systems with Impulsive Effects -State Feedback and Full Information Cases-, Proceedings of the 17th IFAC World Congress, TuA08.4 (CD-ROM), Seoul, Korea, 2008.

[14] G. Nakura: $\mathrm{H}_{\infty}$ Tracking with Preview by Output Feedbak for Linear Systems with Impulsive Effects, Proceedings of the 17th IFAC World Congress, TuA08.5 (CD-ROM), Seoul, Korea, 2008.

[15] Gou Nakura: Stochastic Optimal Tracking with Preview for Linear Continuous-Time Markovian Jump Systems, Proceedings of SICE Annual Conference 2008, 2A09-2 (CD-ROM), Chofu, Tokyo, Japan, 2008.

[16] G. Nakura: $H_{\infty}$ Tracking with Preview for Linear Continuous-Time Markovian Jump Systems, SICE 8th Annual Conference on Control Systems, Kyoto, Japan, 073-2-1 (CD-ROM), 2008.

[17] U. Shaked and C. E. de Souza: Continuous-Time Tracking Problems in an $\mathrm{H}_{\infty}$ Setting: A Game Theory Approach, IEEE Trans. Automat. Contr., Vol. 40, No. 5, pp.841-852, 1995.

[18] C. E. de Souza and M. D. Fragoso: $H^{\infty}$ Control for Linear Systems with Markovian Jumping $\mathrm{Pa}$ rameters, Control-Theory and Advanced Technology, Vol. 9, No. 2, pp. 457-466, 1993.

[19] C. E. de Souza and M. D. Fragoso: $H_{\infty}$ Filtering for Markovian Jump Linear Systems, Int. J. Systems Science, Vol. 33, No. 11, pp. 909-915, 2002.

[20] D. D. Sworder: Feedback Control of a Class of Linear Systems with Jump Parameters; IEEE Trans. Automat. Contr., Vol. AC-14, No. 1, pp. 9-14, 1969.

[21] D. D. Sworder: Control of Jump Parameter Systems with Discontinuous State Trajectories; IEEE Trans. Automat. Contr., Vol. AC-17, No. 5, pp. 740-741, 1972

$$
* * * * * *
$$

This paper is dedicated to Prof. Masao Ikeda, Prof. Akira Ichikawa and Prof. Toshihiro Iwai on the occasions of their sixtieth birthdays. 